Vol. 9 (4): 641-646 (2019)

\title{
FEASIBILITY OF CLEAR-UP STRIPS IN THE DIAGNOSIS OF DEMODEX AND SARCOPTES MITES
}

\author{
Adnan Ayan ${ }^{1 *}$, Songül Erdoğan², Kerem Ural ${ }^{2}$, Hasan Erdoğan², Gizem Gül² \\ ${ }^{I}$ Department of Genetics, Faculty of Veterinary Medicine, Van Yuzuncu Yil University, Tusba, Van, Turkey; \\ ${ }^{2}$ Department of Internal Medicine, Faculty of Veterinary Medicine, Adnan Menderes University, Isikli, Aydin, \\ Turkey; \\ "Corresponding Author: Adnan AYAN, E-mail: adnanayan@yyu.edu.tr;
}

Received August 2019; Accepted September 2019; Published October 2019;

DOI: https://doi.org/10.31407/ijees9407

\begin{abstract}
In the present study, it was aimed to compare the atraumatic Nivea nasal clear-up (NNC) band and traumatic deep skin scraping test (DSST) for the diagnosis of Demodex canis or Sarcoptes scabiei in dogs. A total of 12 specimens were collected from six dogs diagnosed with demodicosis $(n=5)$ or sarcoptic mange infestation $(n=1)$ between September-December 2016 with both techniques. The total number of mites in demodectic dogs with two different methods (NNC) band and DSST were 286 and 159, respectively. In addition, only four eggs were identified with DSST, while 16 adult mites and eight eggs were identified with (NNC) band in a dog with sarcoptic mange. When both technical comparative interpretations are made, it can be said that (NNC) band might be a method which can easily be used in the diagnosis of both diseases and might be used more frequently than DSST. In addition, the atraumatic formation of the application may increase satisfaction of the patient owner.
\end{abstract}

Keywords: Acetate strips, deep skin scraping, demodicosis, dog, sarcoptik scabies 